\title{
Gentrificación como política pública en una ciudad provincial. El caso de la ciudad de Leeds en el Reino Unido ${ }^{1}$
}

\author{
Sara González² y Stuart Hodkinson ${ }^{3}$
}

\begin{abstract}
RESUMEN
Este artículo analiza los procesos de gentrificación en una ciudad media del norte de Inglaterra. El caso de Leeds nos ayuda a provincializar el estudio sobre la gentrificación al centrarnos fuera de las grande ciudades del capitalismo global y posicionarnos en una geografía "provincial". Para capturar las especificidades de la gentrificación en esta ciudad a la vez que contextualizarlas en el marco más amplio del neoliberalismo urbano nos detenemos en 4 variables: escala y posicionamiento, visión relacional de ciudades y políticas urbanas, atención al rol del Estado y a la contestación. De este análisis surgen cuatro principales procesos de gentrificación que tienen lugar en Leeds: city living, gentrificación comercial, estudentificación y mixtura social. Nos basamos en una metodología cualitativa de análisis crítico de las políticas del Estado y una trayectoria de investigación-acción con movimientos sociales en la ciudad. Nuestra conclusión es que la gentrificación en Leeds funciona fuertemente como objetivo de la política urbana pero es un fenómeno inacabado, incipiente o incluso a veces fracasado.
\end{abstract}

Palabras clave: Gentrificación, política urbana, ciudad provincial, Inglaterra.

\begin{abstract}
This article analyses gentrification processes in a mid-sized city in Northern England. This case study of Leeds helps us to "provincialize" the study of gentrification by focusing on a city that is not at the center of global capitalism, but rather is a provincial city. Our aim is to capture the specificities of gentrification in this city at the same time as contextualizing them in the wider frame of neoliberal urbanism. To do that we focus on four variables: scale and positioning, relational cities, the role of the State and its oposition. From this analysis we identify four gentrification trends in Leeds: city living, commercial gentrification, studentification and social mixing. We follow a qualitative methodology that looks critically at public policies and an action research approach through our involvement with community groups. Our conclusion is that gentrification in Leeds functions strongly as a policy objective but it is still incipient, unfinished or has failed.
\end{abstract}

Key words: Gentrification, urban policies, provincial city, England.

1 Artículo recibido el 15 de noviembre de 2013, aceptado el 11 de marzo de 2014 y corregido el 16 de junio de 2014.
2 Escuela de Geografía, Universidad de Leeds (Reino Unido). E-mail: s.gonzalez@leeds.ac.uk

3 Escuela de Geografía, Universidad de Leeds (Reino Unido). E-mail: s.n.hodkinson@leeds.ac.uk 
Este artículo analiza ${ }^{4}$ los procesos de gentrificación en una ciudad media del norte de Inglaterra. Nuestro punto de partida para el análisis de la "gentrificación realmente existente" en Leeds sigue el trabajo de Neil Smith; es decir consideramos la gentrificación como un proceso de reestructuración de clase en el espacio urbano fundamentalmente enraizado en las contradicciones de las relaciones sociales capitalistas. Consideramos importante señalar que al enarbolar la bandera marxista no caemos necesariamente en la "trampa marxista" de ver la gentrificación a través de una lente meramente estructural o demasiado centrada en la producción o en lo material. Es demasiado fácil criticar cualquier análisis marxista por su supuesta tendencia a la generalización, estructuralismo o descontextualización. Si entendemos por "capital" una relación social compleja, contradictoria y cambiante y no simplemente una "cosa" rígida y monetaria entonces los horizontes analíticos se expanden. De este modo, entendemos la gentrificación como un fenómeno inherentemente capitalista, basado en la desigualdad de clase, el desarrollo geográfico desigual y las necesidades cambiantes de la acumulación del capital. Por eso estamos de acuerdo con el análisis de Slater (2006: 747) de que "la gentrificación es un proceso multifacético de transformación de clase que es mejor explicar desde un punto de partida holístico". Este punto de partida incluye no solo la relación entre la dicotomía clásica (y ya superada) de producción y consumo, o los roles de los diferentes actores involucrados, sino también la diversidad y complejidad de cómo y por qué la gentrificación se desarrolla en diferentes sitios, contextos y temporalidades, y cómo las distintas escalas de gentrificación se relacionan entre sí. Tenemos que dejar cla-

4 Este artículo está basado en más de 5 años de investigación empírica y activismo en la ciudad por cada uno de los autores. Los datos provienen de varios proyectos financiados: Autonomous Geographies (ESRC), Public Housing Regeneration under the Private Finance Initiative (ESRC), New enclosures: finance capital and the privatisation of housing privatisation in Britain (British Academy) New Urban Deal (Leverhulme Trust) y la participación activa en grupos como Hands of Our Homes Leeds y Friends of Leeds Kirkgate Market. La investigación se enmarca también en el proyecto financiado por la UE Contested Cities (FP7-PEOPLE-PIRSESGA-2012-318944). ro que el retrato original de Glass en 1964 de la gentrificación, como un proceso de rehabilitación y mejora de vivienda degradada en zonas centrales, llevado a cabo por pioneros de clase media desplazando a los residentes de clase trabajadora con la subida de alquileres, ha sido superado. Entendemos que hoy en día la gentrificación está en la punta de lanza del urbanismo neoliberal (Smith, 2002) y se ha convertido en una estrategia urbana global que en muchas ocasiones se ha intensificado con la actual crisis económica global que plaga Europa. Tampoco puede entenderse la gentrificación como un proceso geográficamente restringido a los centros urbanos o las metrópolis del Norte Global. En resumen, siguiendo a Kate Shaw, entendemos la gentrificación como:

“(...) una re-estructuración generalizada del espacio en términos de clase media, que engloba la total transformación de barrios de bajo estatus en patios de recreo de las clases media/altas. Las viviendas de los agentes gentrificadores ya no son solo casas renovadas sino viviendas de nueva construcción y apartamentos (...). La gentrificación se extiende también a los locales comerciales y puede apreciarse tanto en pueblos rurales o de costa como en ciudades (...) (Shaw, 2008: 2).

Nuestra contribución a los debates sobre la gentrificación hace referencia a la importancia del "posicionamiento" y de tener una mirada "relacional" cuando se analizan procesos de gentrificación. Los trabajos sobre gentrificación más reciente hacen un Ilamamiento por "descentralizar" los estudios de gentrificación y no asumir que lo que ocurre en ciudades americanas o británicas es generalizable a otra ciudades del mundo (Janoschka et al., 2014; Lees, 2012). En concreto, se habla de la necesidad de enfatizar las diferencias con respecto al "centro neurálgico anglófono" (Maloutas, 2011). Pero es necesario también reflexionar críticamente sobre este "centro" ya que tampoco se puede generalizar que todo lo que ocurre en las ciudades "anglosajonas" sea lo mismo. Londres, el epicentro conceptual del término gentrificación, es por ejemplo una ciudad muy diferente de Leeds. Tampoco se puede generalizar hacia dentro de las ciudades: ¿todos los cambios urbanos en una ciudad son gentrificación, 
o hay diferentes causas, procesos, etcétera, que diferencian lo que ocurre en una barrio de otro? Muchas veces, como lo ha apuntado Robinson (2005), son las ciudades más grandes como Nueva York o Londres las que parecen marcar la agenda de los estudios urbanos y se pasa de largo lo que ocurre en ciudades más pequeñas y de "segundo orden". Este es el caso de la ciudad de Leeds, una ciudad de tamaño mediano según los estándares europeos, en el norte del Reino Unido. Los procesos de gentrificación que tienen lugar en Leeds no son simplemente una repetición de lo que ocurre en Londres, hay muchas diferencias aunque también similitudes.

Respondiendo a Maloutas (2011), el hecho que creamos todavía en el valor analítico, crítico y político del término gentrificación y que nos interesemos por las diferencias y similitudes entre ciudades no quiere decir que no seamos muy conscientes del contexto local y de los procesos y mecanismos causales. Nos situamos en el horizonte teórico trazado por Brenner et al. (2010) y su concepto de "variegated neoliberalism" que alude la necesidad de prestar atención tanto a las estructuras institucionales más amplias y sólidas como a los experimentos que tienen lugar en territorios específicos pasando por el fenómeno de la transferencia internacional de ideas y políticas. Siguiendo esta trayectoria teórica en este artículo, también seguimos y nos referimos a los tipos y dimensiones de gentrificación que Janoschka et al. (2014) identifican y notamos las diferencias y similitudes con otras ciudades. En el caso de Leeds nos centramos en 4 procesos de gentrificación que tienen lugar a la vez pero con escalas temporales y geográficas particulares y que hemos denominado, mediante el (ab)uso de anglicismos: city living, gentrificación comercial, estudentificación y mixtura social. Antes de analizar en detalle estos procesos nos detenemos en una rápida introducción a la ciudad de Leeds y en una reflexión teórica sobre el estudio de la gentrificación en una ciudad "provincial".

\section{Leeds: una breve guía al "Londres del norte"}

Leeds es una ciudad del norte de Inglaterra con una población en crecimiento de
751.500 personas que viven en 320.600 hogares de acuerdo con el último censo del 2011 en sus fronteras municipales y una población de alrededor de 3 millones en la ciudad-región más amplia. Es la ciudad más grande y rica de la región de Yorkshire (donde se encuentra también Sheffield), una de las regiones más pobres de Inglaterra a su vez en una de las naciones más divididas de Europa, con un norte pobre y un sur (con Londres a la cabeza) más rico (González, 2011). Esta división regional se ha acrecentado con la crisis financiera desde el 2008 (London Economics, 2013). Leeds es además una ciudad diversa desde el punto de vista económico, cultural y étnico; el $11 \%$ de la población proviene de minorías étnicas. Históricamente Leeds fue un centro textil, comercial y manufacturero pero un proceso de desindustrialización a nivel nacional desde los años 70 ha ido convirtiendo la base económica de la ciudad hacia los servicios. En el año 2011, un 86\% de los empleos en Leeds eran en pequeñas empresas en el sector servicios (Leeds City Council, 2013b). Leeds se considera una ciudad con una economía dinámica y diversa y con menos empleos en el sector público que otras ciudades del norte de Inglaterra, más tradicionalmente "dependientes" del Estado central (Centre for Cities, 2011). El PIB per cápita de la ciudad-región de Leeds representa un 95\% de la media Europea, lo que es similar a otras grandes ciudades británicas con excepción de Londres (Eurostat, 2013).

Dentro del sector servicios, la economía de Leeds se ha expandido particularmente hacia los servicios financieros, catapultando Leeds a ser el centro financiero de más crecimiento en el Reino Unido fuera de Londres (Tickell, 1996: 103) y dando alas a los líderes políticos de Leeds para vender su ciudad como una "ciudad de finanzas". El crecimiento de este sector es en parte debido a la descentralización geográfica de oficinas con base en Londres pero también hay una tradición histórica de pequeñas cajas de ahorro. La especialidad en el sector financiero en Leeds son los aspectos legales de las finanzas, hipotecas, finanzas comerciales y call centres (Henderson, 2005).

Además del sector financiero, otro de los pilares más importantes de la economía de Leeds ha sido el boom inmobiliario en el 
centro de la ciudad. Como otras ciudades británicas, Leeds ha promovido en las últimas décadas lo que en el Reino Unido se Ilama "city living"; es decir "vivir en el centro". Los centros de la mayoría de las grandes ciudades británicas, a excepción de Londres y ciudades escocesas como Glasgow y Edimburgo, han carecido históricamente de densidad residencial pero una política de "vuelta al centro" más el boom de la construcción desde principios de los 2000 ha conllevado la construcción de nuevos bloques de viviendas en el centro de Leeds (ver más adelante). Además de viviendas residenciales, a mediados de los 2000, Leeds planeaba innumerables proyectos urbanísticos de alto valor simbólico todos en el centro de la ciudad que le valieron el pseudónimo de "ciudad rascacielos" (Chatterton \& Hodkinson, 2007). Otro de los pilares de la estrategia de acumulación en Leeds ha sido el comercio y la atracción al centro de la ciudad de más cadenas internacionales para convertir a Leeds en un "destino de compras" y escalar el ranking nacional de comercio por encima de Manchester (Locate in Leeds, 2012). Esta regeneración urbana del centro de Leeds con nuevos apartamentos, bares de moda y tiendas de diseño es lo que le ha valido a Leeds el apodo de "Londres del Norte".

Pero lejos de lo que los líderes locales consideran una "historia de éxito" de Leeds, la ciudad es una urbe dividida. El 19\% de las unidades "super output ${ }^{5}$ " censales en Leeds se encuentran en el $10 \%$ de las más pobres a nivel nacional (Leeds City Council, 2013b). Es además una ciudad segregada espacialmente donde la mayor parte de la población de clase baja y proveniente de minorías étnicas reside en la "inner city", es decir zonas de alta densidad urbana al borde del centro, y en el sur-este de la ciudad mientras que las zonas residenciales de norte concentran residentes de clase media-alta. Es importante remarcar, sin embargo, que esta segregación espacial no llega a los límites de otras ciudades sobre todo en el sur global como las que

\footnotetext{
5 Esta es una unidad geográfica estadística desarrollada por la Oficina de Estadísticas Británica (ONS) que contiene unas 1.500 personas. Inglaterra contiene $32.484 \mathrm{SOAs}$, de las cuales 476 están en Leeds.
}

se tratan en este número especial. No existen prácticamente comunidades y barrios enrejados, slums (villas, favelas, etcétera) o guetos a la americana.

En cuanto al gobierno y la política local, Leeds ha mantenido históricamente un perfil más modesto que otras ciudades como Manchester, que siempre se considera como una ciudad con más espíritu emprendedor y más dispuesta a "venderse" mediante estrategias de marketing urbano (Haughton \& Williams, 1996; Ward, 2003). En Leeds, como en la mayoría de las grandes ciudades británicas, existe una tradición colaborativa entre el sector privado y público, aunque de menor calado que en ciudades como Manchester. Sucesivos gobiernos centrales han promovido el estrechamiento de los lazos entre las autoridades locales y los líderes empresariales mediante financiación de partenariados, proyectos de regeneración, redes y organizaciones. El presente gobierno conservador-liberal ha reforzado esta trayectoria imponiendo la formación de redes de gobernanza local relativamente sólidas que combinan el sector privado y público. En Leeds existe una coalición público-privada a nivel metropolitano (Leeds city-region) que ha ido adoptando cada vez más responsabilidades sobre todo en el plano urbanístico y de empleo.

\section{La gentrificación en una ciudad "provincial"}

Diversas tendencias recientes en la geografía crítica han llamado a la "provincialización" del término gentrificación. Por un lado, "provincializar" significa reconocer que los contornos y características de la gentrificación están apegados al momento y geografía específica donde el término se acuñó; el Londres de los años 60. Es decir, no son características universales sino histórica y geográficamente específicas. Sheppard et al. (2013: 4) argumentan que la "provincialización es una estrategia crítica a través de la cual lo "universal" se revela simplemente como un simple contenedor (place-holder en inglés)". En los estudios urbanos, Jennifer Robinson (2005) ha promovido esta agenda por "descentrar" la academia anglosajona de su tendencia a favorecer el estudio de las "ciudades globales", que están en el centro de las 
redes de flujo del capital financiero internacional. En contraste, Robinson Ilama a ocuparse también de la ciudad ordinaria. Harris (2008), por ejemplo, afirma que hay mucho que aprender de los procesos de gentrificación y contestación urbana que tienen lugar en ciudades fuera del epicentro neoliberal.

Por otro lado, en el caso del Reino Unido el uso del término "provincial" tiene un significado diferente aunque relacionado. Se utiliza por autores británicos para referirse a los procesos de gentrificación fuera de Londres (Smith \& Holt, 2007; Boddy, 2007). Se trata de entender si los mismos procesos de gentrificación que tienen lugar en Londres ocurren en ciudades fuera de la capital, o si estos son específicos a Londres por tratarse de una ciudad global. Por ejemplo, Bridge (2002) realiza un análisis de las trayectorias residenciales de familias en Bristol. Su conclusión es que es más fácil mantener trayectorias residenciales gentrificadoras en Londres porque el parque de viviendas con potencial gentrificador es más amplio y puede acomodar los cambios en los gustos, estilos de vida y necesidades de los gentrificadores. El análisis de Dutton (2008) en Leeds también arroja esta misma conclusión que se generaliza a varias ciudades provinciales. Sin embargo otras características de la gentrificación en Leeds, de acuerdo con Dutton (2008), no tienen que ver con su carácter provincial sino con condiciones locales.

El caso de Leeds es interesante entonces, porque aunque se trata de una ciudad que inicialmente (sobre todo al compararla con las otras ciudades de este dossier) podría verse en el centro neurálgico del neoliberalismo global, es también una ciudad "provincial". De forma modesta argumentamos que no solo podemos "provincializar" lo que sabemos sobre la gentrificación desde una mirada desde el sur global sino también desde una ciudad secundaria del norte de Inglaterra.

Este artículo también contribuye al incipiente grupo de trabajos sobre los procesos de gentrificación en Leeds. Haciendo una revisión bibliográfica encontramos tres tipos de estudios: En primer lugar, un trabajo que analiza de forma explícita y crítica los procesos de gentrficación específicos en Leeds (Dutton, 2003, 2008; González \& Waley,
2013; Green, 2007; Hodkinson, 2011); en segundo lugar, trabajos que se centran en la regeneración urbana que ha tenido lugar en el centro de Leeds en los últimos años y sobre todo en el boom residencial. Estos estudios están escritos desde una perspectiva del profesional urbanista y menos críticos (Nathan \& Unsworth, 2006; Stillwell \& Unsworth, 2008; Unsworth, 2007; Unsworth \& Smales, 2010); y en tercer lugar, una colección de estudios más general y diversa que discute varios aspectos de la vida urbana y política en Leeds y otras ciudades similares y que discute temas relacionados con la gentrificación desde una variedad de perspectivas analíticas (Chatterton \& Hodkinson 2007; Hodkinson \& Chatterton, 2007; Julier, 2011; Smith, 2002, 2005, 2007, 2008). Además de énfasis en el fenómeno de "city living" (ver más adelante) también se presta atención en estos estudios a los cambios en la cultura urbana y las prácticas de consumo (Bell, 2009; Harcup, 2000; Chatterton \& Unsworth, 2004; Spracklen et al., 2013), el rol del marketing de ciudades (Julier, 2011; O'Connor et al., 2008), la cuestionable calidad de los nuevos paisajes urbanos en el centro (Haughton, 2009) y el efecto gentrificador del Ilamado fenómeno de la estudentificación (ver más adelante) (Chatterton, 2010; Smith, 2002, 2007).

Sobre la base de esta literatura que ya ha analizado diversos procesos urbanos en Leeds, nuestra contribución tiene como objetivo examinar de forma global la política urbana y los cambios en la ciudad de las últimas décadas bajo la óptica de la gentrificación. Combinando los dos significados ya mencionados del término "provincial" proponemos que para entender el tipo de gentrificación que encontramos en Leeds tenemos que considerar cuatro variables. Estas variables nos ayudan a contextualizar la gentrificación a la vez que no nos alejan de una mirada más amplia que busca similitudes $y$ diferencias.

\section{Escala y posicionalidad}

Posicionalidad se refiere a "la forma de capturar los modos cambiantes, asimétricos pero dependientes de trayectoria (pathdependent) en los que el futuro de un lugar depende de las interdependencias con otro lugar" (Sheppard, 2002: 308). Prestar aten- 
ción a la "posicionalidad", de acuerdo con Sheppard significa analizar las conexiones entre lugares (cercanos, lejanos y aparentemente desconectados) y cómo estas conexiones pueden dar lugar a desigualdades en la economía global. Significa prestar atención a las escalas geográficas, que no son contenedores herméticos organizados de forma jerárquica desde la escala local a la global pasando por la nacional, sino constructos sociales y políticos que entrañan relaciones desiguales de poder (González, 2005). Así, tenemos que contextualizar a Leeds desde diversas posicionalidades que ayudan a explicar el tipo de gentrificación que podemos encontrar en esta ciudad: como una ciudad de tamaño medio y de rango secundario a nivel Europeo e internacional; como una ciudad "provincial" del norte en un país extremadamente centralizado dominado por la órbita londinense y con una significativa disparidad económica regional entre el norte y el sur del país; como la ciudad central de una región urbana desigual con ciudades, como Bradford, que tienen dificultades en atraer inversiones y altas tasas de pobreza.

\section{Ciudades en relación y políticas móviles}

Cada vez hay más necesidad de entender las ciudades de forma relacional (Ward, 2010), es decir, no como realidades que empiezan y acaban en unos límites geográficos administrativos sino más como nudos o centros de flujos que se extienden más allá de estas fronteras. Estos flujos pueden ser tanto materiales (ciertas mercancías, capital) como, cada vez más, ideas, símbolos, políticas, etc. Las políticas urbanas que pueden conllevar a la gentrificación de las ciudades también viajan (Peck \& Theodore, 2010) y al viajar se transforman, mutan y adaptan a las especificidades locales (González, 2011). Es interesante analizar desde qué fuentes beben los políticos y funcionarios locales para entender mejor cómo y por qué se implementan políticas gentrificadoras. La ciudad de Leeds está unida con otras ciudades por varias redes como por ejemplo Eurocities, donde se intercambian "recetas de éxito" entre ciudades europeas, y está unida por acuerdos bilaterales con varias otras ciudades. En Leeds, por ejemplo, Lille y Barcelona son a menudo abanderadas como modelos de éxito de regeneración urbana. En el 2010 y 2011 el ayuntamiento de Leeds en colaboración con otros agentes privados organizó sendas "expediciones" a Barcelona. La mención de supuestas "recetas de éxito" de políticas urbanas en lugares lejanos de los que es difícil tener una mirada crítica es un mecanismo poderoso de justificación y despolitización del proceso de decisión político. Es importante por lo tanto desmadejar los flujos que traen y llevan ideas de intervenciones urbanas para poder tener una perspectiva más crítica de la gentrificación.

\section{El rol del Estado central}

En el caso de Leeds, una ciudad británica, es importantísimo tener presente el rol del Estado. Si bien quizá en Latinoamérica y en menor medida en España el Estado ha jugado tradicionalmente un papel menor, en el Reino Unido el Estado es un agente principal a la hora de analizar la gentrificación urbana. En el Reino Unido podemos analizar las últimas décadas de políticas urbanas desde el marco planteado por Brenner y Theodore (2002) de roll-back y roll-out, que podríamos traducir como retroceso y despliegue del Estado. Según este análisis el Estado, bajo el gobierno de Thatcher en el período 1979-1997 se retrajo dejando más espacio a la iniciativa privada para gestionar las ciudades. Se desmantelaron las grandes corporaciones metropolitanas como la de Leeds y su región (dominadas políticamente por la izquierda) y estructuras nacionales del Estado de Bienestar y en su favor se establecieron nuevos partenariados locales en el ámbito del desarrollo local que favorecían los intereses privados. Se acrecentó también la competición interurbana, al inaugurar la competición entre ciudades por fondos públicos para la regeneración urbana y lucha contra la pobreza (Jones \& Ward, 2002). Así, ya no era el gobierno central quien determinaba la distribución de fondos en base a la necesidad con el objetivo de estandarizar las condiciones de vida a nivel nacional, sino que se abría la veda para la promoción de las disparidades entre ciudades. Además se siguió un modelo de regeneración urbana centrado en la especulación sobre todo de las zonas centrales de las ciudades o en lo que se llaman las "inner cities", zonas pobres adyacentes al centro donde existían áreas ex industriales inutilizadas. A esta mercantilización del espacio urbano se sumó el 
abandono de la política social de vivienda lo que promocionó la gentrificación de zonas urbanas centrales sobre todo en Londres. La siguiente oleada de regeneración urbana nos lleva a la etapa de "roll out", o despliegue de un nuevo tipo de Estado bajo el gobierno laborista de Tony Blair que Ilegó en 1997. En esta nueva etapa, el énfasis se puso en el concepto de "comunidad", "inclusión social" y la participación de la sociedad civil en las políticas de desarrollo. El eslogan de la política urbana fue el "renacimiento urbano" y el objetivo revitalizar los centros urbanos dotándolos de vida y actividad comercial. Además se puso freno al desarrollo suburbano de proyectos residenciales y de centros comerciales que se había desplegado con el gobierno de Thatcher y se promocionó la construcción de vivienda en el centro de las ciudades. En realidad, en términos materiales, la política urbana laborista fue una continuación de la conservadora, confiando en la inversión privada en centros urbanos y vivienda privada que después pudiera, indirectamente, revitalizar las economías urbanas y contribuir a la reducción de la pobreza.

Lo que queremos destacar es que en el Reino Unido, no es el vacío del Estado lo que provoca la acción de los actores privados en el desarrollo urbano, sino la propia actividad del Estado la que promociona políticas gentrificadoras (state-led gentrification).

\section{Contestación}

La contestación a las políticas urbanas gentrificadoras es también una variable indispensable a la hora de analizar la gentrificación. Desde una perspectiva autonomista, la resistencia de los trabajadores se comprende como parte de la relación social capitalista. Los trabajadores tienen autonomía para organizarse en contra del capital, y este se adapta, moldea y transforma en respuesta. La evolución del capitalismo no está solo motivada por cambios tecnológico sino por la autonomía de los trabajadores. Si extendemos este análisis autonomista a los fenómenos urbanos observamos que en muchas ocasiones las políticas de gentrificación se encuentran con la fuerza subversiva de la ciudadanía que las contesta y opone. No existe sin embargo un cuerpo considerable en la literatura anglosajona que teorice cómo la resistencia, la contestación y los movimientos sociales urbanos ponen límites, modifican o consiguen frenar la gentrificación. Pero como apuntan Janoschka et al. (2014) la literatura en español sobre el tema ha dedicado mucho más espacio al rol de la resistencia urbana. Así como la gentrificación se ha convertido en una estrategia urbana global (Smith, 2002), también la contestación ha adquirido dimensiones globales sobre todo desde la emergencia de la crisis financiera global. En multitud de ciudades desde Estambul a Río han surgido movimientos en contra de megaeventos y megaproyectos urbanos que desplazan a los más pobres o en contra del urbanismo de austeridad y recortes de las políticas sociales que afectan a los más vulnerables en nuestras ciudades. En contraste con lo que anotan Janoschka et al. (2014) en el caso de Madrid y ciudades latinoamericanas en Leeds no hay una tradición muy fuerte de movimientos sociales urbanos aunque sí encontramos prácticas de resistencia urbana a varios niveles que comentaremos en nuestro análisis más específico de la gentrificación más abajo.

\section{Cuatro procesos de gentrificación en la ciudad de Leeds}

En esta sección nos centramos en la parte más empírica de nuestro análisis y desarrollamos los principales 4 procesos de gentrificación que están teniendo lugar en Leeds y que denominamos con el uso de neologismos y/o anglicismos: "city living", "gentrificación comercial", "estudentificación" y "mixtura social"

\section{City living}

Aunque el centro urbano de Leeds estaba poblado densamente en parte por asentamientos muy pobres (casi slums) en el siglo XIX, esta población fue paulatinamente desplazada hacia complejos residenciales en las zonas adyacentes al centro (inner city) o en la periferia a lo largo del siglo XX. Así, los centros urbanos de las ciudades británicas (menos en los casos de Londres y quizá Edimburgo y Glasgow) han carecido de una densidad residencial a diferencia de la Europa continental urbana. En el Reino Unido, el hogar típico es generalmente una casa unifamiliar con jardín privado en un barrio residencial y la tradición 
urbanística no ha promocionado la mezcla de usos del suelo. Sin embargo, como hemos dicho antes, desde finales de 1990 se lanzó una estrategia nacional de "renacimiento urbano" (urban renaissance) para combatir el declive de los centros urbanos que habían sido diezmados por la política de Thatcher de promoción grandes superficies comerciales en las afueras de las ciudades con libre aparcamiento. Además, durante décadas, sobre todo en las década de 1970 y 1980, los centros urbanos se veían como problemas, zonas de concentración geográfica de crimen $y$ tensiones raciales e incluso hubo erupciones violentas que terminaron con enfrentamientos con la policía en varias ciudades incluyendo Leeds. El nuevo impulso desde el gobierno era una oportunidad para "recuperar" estos espacios urbanos para el consumo, la vivienda y el disfrute de las clases medias. El "nuevo laborismo" de Tony Blair en 1997 persiguió transformar los centros urbanos en nuevas áreas de consumo y cambiar su imagen de problemas urbanos a oportunidades. Ciudades como Barcelona y otras del continente europeo se utilizaron como modelos de esta nueva densidad residencial.

En Leeds, una primera fase de este proceso de "renacimiento urbano" tuvo lugar entre mediados de las décadas de 1980 y 1990, con la reconversión de antiguos edificios industriales y fábricas textiles en condominios más algunos bloques de nueva construcción. Este tipo de nuevo estilo de vida urbano respondía a la demanda de capital cultural y estético de las nuevas clases profesionales dedicadas al negocio financiero, parte de las cuales se había transferido desde Londres (Dutton, 2003). Desde finales de los 90 hasta mediados de los 2000 esta renovación del centro urbano entra en una etapa de especulación inmobiliaria frenética. Entre 2003 y 2009 se construyeron en Leeds 9.500 apartamentos en el centro pero existía licencia urbanística para la construcción de muchos más (Unsworth, 2010). Estos proyectos fueron construidos por lo que Chatterton y Hodkinson (2007) Ilamaron los "5 grandes"; 5 empresas promotoras locales o regionales que se repartieron todos los contratos. El capital provino, en parte, de inversores desde Irlanda o el Medio Oriente que buscaban donde invertir después de la caída de la bolsa como consecuencia de los atentados del 11 de septiembre de 2001. Muchas de estas operaciones eran de compra para alquiler por propietarios que nunca habían estado en Leeds y muchas veces los apartamentos permanecían vacíos esperando a su revalorización. Este boom inmobiliario también fue fomentado, en parte, por una política macroeconómica de mantenimiento de intereses bajos fomentando los créditos inmobiliarios y la especulación urbanística.

Estos apartamentos se construyeron sin la exigencia de un plan estratégico del gobierno local, quien aprobó licencias urbanísticas ad hoc. Esta "ausencia" intencionada del gobierno local en el terreno de planificación urbana puede analizarse como parte de una estrategia de gentrificación inducida por el Estado (state-led gentrification) que toma una decisión de abrir las puertas a proyectos residenciales que podríamos Ilamar elitistas sin preocuparse por el hecho de que muchos ciudadanos en Leeds no se puedan permitir vivir en ellos.

Los esfuerzos por promover el centro de la ciudad como una nueva zona residencial y de ocio han sido claramente dirigidos a una población de clase media/alta con alto poder adquisitivo. Existe en la literatura académica un debate sobre si este tipo de nuevas formas residenciales pueden analizarse como "gentrificación de nueva construcción" (new build gentrification) ya que algunos autores argumentan que no desplazan directamente a población (Lambert \& Boddy, 2002 citado en Lees \& Davidson, 2010). Sin embargo, el trabajo de Lees y Davidson (2010) ha mostrado que basándose en las categorías de Marcuse $^{6}$ (1985) podemos encontrar procesos difuminados de desplazamiento también en este tipo de proyectos residenciales de nueva construcción. En Leeds, claramente se da una situación de "desplazamiento ex-

\footnotetext{
6 1) Desplazamiento directo del último residente de una vivienda; 2) cadena de desplazamientos directos teniendo en cuenta previos residentes; 3) desplazamiento excluyente (aquellos que no acceden a la vivienda porque el área ya está gentrificada) y 4) desplazamiento por presión, cuando el desplazamiento no es directo o forzado sino por la presión que ejercen las transformaciones urbanas (falta de transporte público, subida de precios de los servicios, cambio del vecindario)
} 
cluyente" (ver nota 6), ya que los residentes de menor poder adquisitivo que se podrían beneficiar de una vivienda en el centro de la ciudad con acceso a puestos de trabajo, no podrían permitirse los precios. Otra dimensión gentrificadora es el hecho de que estos proyectos orientados a una clase media han revalorizado el centro de la ciudad como zona residencial lo que a su vez pone en valor las zonas adyacentes al centro, por su accesibilidad, que como ya hemos explicado son tradicionalmente de clase trabajadora y tienen precios más bajos. Así han surgido en Leeds varios proyectos de reconversión de vivienda en las zonas adyacentes al centro que tienen el efecto de subir los precios del suelo. Además estos nuevos proyectos residenciales han contribuido a cambios en el paisaje comercial de la ciudad con la emergencia de una multitud de supermercados que antes no existían y nuevos centros comerciales (ver más abajo) que compiten con los comerciantes independientes.

Por último y siguiendo la tipología de Janoschka et al. (2014), en términos de gentrificación simbólica, estos nuevos bloques de apartamentos han gentrificado el perfil urbano de la ciudad ya que antes de su existencia la mayoría de los bloques de apartamentos en Leeds eran de vivienda social que según el propio ayuntamiento "afeaban" el paisaje urbano "dañando el carácter de la ciudad" (Leeds City Council, 2007: 34). La concentración de nuevos bloques residenciales y edificios altos de oficinas en el centro se interpretan como una mejora de la imagen de Leeds como ciudad europea competitiva.

No ha habido prácticamente ningún tipo de contestación ciudadana a estos cambios radicales del centro de la ciudad, lo que en parte se puede explicar por la falta más generalizada de una arena de debate local en un país centralizado. Sin embargo sí ha habido críticas por parte de académicos y arquitectos de la ciudad y a nivel nacional por la falta de sostenibilidad de estas nuevas formas residenciales que se concentran en un sector de la población muy determinado y no "crean" ciudad (Haughton, 2009). Un arquitecto de renombre incluso comentó (BBC, 2009) que estos apartamentos se podrían convertir en una especie de "favelas del futuro". Desde la llegada de la crisis financiera global en el 2008 muchos de los proyectos de nuevos bloques de apartamentos se pararon y por lo tanto no se llegaron a construir.

\section{Gentrificación comercial}

El centro de la ciudad de Leeds es relativamente pequeño. Mientras que todo el municipio de Leeds cubre 551 kilómetros cuadrados, el centro se extiende tan solo 4,6 $\mathrm{km}^{2}$ (Leeds City Council, 2013a). Este pequeño centro está mayoritariamente orientado al comercio y consumo con 957 tiendas en lo que se conoce como el distrito comercial (Ibid). Leeds es el séptimo distrito comercial más grande del Reino Unido y el gobierno local considera uno de sus objetivos principales el promocionar la ciudad en los rankings comerciales y escalar puestos en la jerarquía urbana sobre todo por delante de su rival histórico Manchester. Este distrito comercial tiene tiendas de lujo y diseño comparables a Londres y cuenta con un nuevo centro comercial Ilamado Trinity, el más grande en Europa Occidental de su tipo (Kollewe, 2012). Pero también podemos encontrar innumerables pequeños comercios independientes y uno de los mercados de abasto más grandes de Europa con cientos de puestos muchos de ellos todavía gestionados en familia. Se trata por lo tanto de un distrito comercial diverso pero los recientes cambios en el centro urbano lo empujan a una situación de gentrificación.

Leeds es una de las pocas grandes ciudades del Reino Unido que, en tiempos de recesión económica y austeridad, ha conseguido consolidar planes para dos centros comerciales en el centro de la ciudad: Trinity y Victoria Gate. Los promotores de estos dos centros comerciales se encuentran entre los más grandes inversores comerciales e inmobiliarios del Reino Unido y Europa (Land Securities y Hammerson). Aunque se trata de inversiones privadas, el ayuntamiento ha contribuido con recursos propios, personal, promoción y facilidades para su llegada y establecimiento. De hecho se promocionan por el ayuntamiento como los proyectos más importantes de la ciudad en este momento (además de un auditorio de música). Trinity abrió sus puertas en abril del 2013 y se puede ver claramente que el paisaje comercial ha cambiado a su alrededor. Han desaparecido comercios, bares y restaurantes que Ilevaban 
en la zona muchos años y reemplazados por tiendas pertenecientes a cadenas nacionales o multinacionales o comercios dirigidos a clientela de mayor poder adquisitivo. Merece la pena concentrarse en el segundo de los centros comerciales, Victoria Gate, que empezó a construirse en abril del 2014 y que ya se anuncia como un destino de compras de lujo. El centro se construirá en una zona de la ciudad que ha sido activamente abandonada por el ayuntamiento que desde décadas espera una inversión privada que no llegaba. Desde nuestra aproximación teórica entendemos que la desinversión y el abandono planificado son fases previas a la gentrificación. El ayuntamiento designó esta zona a principios de los años 2000 como zona de expansión del distrito comercial pero no se materializó ninguna inversión privada y menos desde la crisis económica del 2008 hasta que Hammerson en 2011 hizo una propuesta firme para levantar un centro comercial con un gran casino. La zona ha sufrido un declive urbano desde los años 70, cuando la carretera de circunvalación de la ciudad creó una barrera y tanto los concejales y funcionarios como promotores privados insisten en referirse a la zona hoy como "vacía". Sin embargo contiene dos grandes aparcamientos propiedad del ayuntamiento (que sirven al mercado adyacente), algunas viviendas, comercios y lo que era el embrión del China Town de Leeds que poco a poco ha sido destruido para hacer sitio para el nuevo centro comercial.

Toda esta zona alrededor de este futuro centro comercial y el mercado de abasto del que hablaremos más abajo, se considera por el ayuntamiento como "la pieza que falta en la regeneración del centro de la ciudad" (Leed City Council, 2011a). Es una zona de comercios independientes con una mezcla particular de tiendas de ropa de segunda mano, peluquerías y tiendas de muebles de bajo coste, estudios de tatuajes y establecimientos de comida rápida. Además se localizan algunos de los edificios más antiguos de la ciudad, donde se compraban y vendían textiles en el s.19, y que se encontraban casi en estado de ruina (Baron, 2010a). Toda esta zona se puede analizar como "en espera", en la antesala, en la frontera urbana de la gentrificación, con una mezcla de desinversión, edificios y locales desatendidos, comercios de bajo valor añadido, una clientela de bajo poder adquisitivo pero a la vez "potencial gentrificador" por su centralidad estratégica entre dos nuevos centros comerciales y valor arquitectónico de algunos de los edificios. También, en esta zona encontramos lo que podría llamarse un proceso de gentrificación comercial fallido. El Corn Exchange es un edificio con alto valor patrimonial que desde mediados de los 80 se utilizaba como una especie de centro comercial independiente que atraía a jóvenes goth y emo de todos los alrededores de Leeds. En el 2007 el propietario del edificio, la compañía global aseguradora Zurich rompió los contratos con los comerciantes y los desahució como parte de un plan para convertir el edificio en una especie de "centro gourmet internacional", en contra de la opinión pública y protestas de jóvenes (Leeming, 2008). Sin embargo la estrategia fracasó y no se atrajeron suficientes inversores para llenar el edifico que todavía permanece medio vacío. Podemos ver el caso del Corn Exchange como un recordatorio de que los planes de gentrificación no siempre tienen éxito. Las ambiciones del ayuntamiento de Leeds son convertir el distrito comercial en una zona sanitizada que atraiga a consumidores de alto poder adquisitivo de Leeds y de toda la región, pero la realidad es que Leeds es una ciudad socialmente polarizada que quizá no tiene una masa crítica enorme de población de clase media alta que se pueda permitir consumir es estos espacios y además los inversores nacionales e internacionales están más interesados en ciudades más grandes como Londres o Manchester. La condición de ciudad norteña provincial pone límites a los sueños gentrificadores de las élites locales.

También en esta zona "atrapada" en la frontera urbana de la gentrificación se sitúa uno de los mercados de abasto más grandes de Europa, el Kirkgate Market. Hemos analizados la situación de este mercado con más detalle en otra publicación (González y Waley, 2013) pero vale decir que concentra las mismas características que hemos descrito de la zona más amplia más arriba: décadas de desinversión y abandono municipal, comercios pequeños que funcionan en una lógica de bajo valor añadido y con predominio de clientela de bajo poder adquisitivo. Con los planes de Victoria Gate, el ayuntamiento y el promotor privado Hammerson se han 
interesado por el potencial valor estratégico de este mercado que según su razonamiento necesita incorporarse en una nueva lógica comercial más en sintonía con el regenerado distrito comercial y atraer a una clientela más acaudalada vendiendo productos diferentes y "modernizando" (según esta lógica) la estética de sus puestos y mercancía.

Desde el 2010 ha surgido un grupo de ciudadanos y usuarios del mercado Ilamado Friends of Leeds Kirkgate Market que ha contestado directamente estos planes potencialmente gentrificadores y ha defendido la importancia del mercado como espacio público por excelencia en el centro de Leeds del que dependen los ciudadanos más pobres por sus precios asequibles (Friends of Kirkgate Market blog). Este grupo se ha convertido en un interlocutor con las autoridades locales y ha conseguido promover el tema del futuro del mercado en el debate local, cuestionando el discurso dominante. Aunque no ha conseguido parar los planes de reconversión del mercado sí ha generado una solidaridad con los comerciantes y un discurso alternativo bastante compartido por la población en general.

\section{Estudentificación}

Estudentificación, es un anglicismo que se refiere al proceso por el que los estudiantes universitarios "toman" un espacio urbano o también el proceso por el que el estilo de vida estudiantil tiende a dominar el paisaje comercial, residencial, cultural y servicios (Smith \& Holt, 2007). Leeds cuenta con dos grandes universidades que atraen casi a 60.000 estudiantes. Los dos campus se sitúan al norte del centro y del distrito comercial lindando con las zonas más residenciales de la "inner city" y barrios más burgueses que han sufrido grandes cambios residenciales. Según nuestro análisis podemos identificar dos oleadas de estudentificación de Leeds en los últimos 15 años. La primera fase coincidió con la expansión de la educación universitaria bajo el gobierno de Blair y un proceso más amplio de profesionalización y tercerización postindustrial de la economía británica que sobrepasó la oferta de las tradicionales residencias de estudiantes. Hay que añadir también que la realidad británica es particular porque la mayoría de los estudiantes universitarios buscan universidades lejos de la residencia de sus padres lo que obliga a la provisión de vivienda estudiantil. El crecimiento del número de estudiantes, entonces, estimuló un proceso de especulación inmobiliaria en el que la iniciativa privada, con prácticamente ningún control urbanístico, compró viviendas grandes unifamiliares en zonas de bajo precio y las reconvirtió en viviendas de alquiler con habitaciones para estudiantes extrayendo grandes cantidades de renta. El proceso de estudentificación no ha sido total en estos barrios ya que existen áreas de vivienda social y familias establecidas sobre todo de origen asiático pero el paisaje residencial ha cambiado con una gran densificación que afecta a la provisión de servicios como la recogida de basura. La Ilegada de estos estudiantes de clase y origen étnico diferente a la población establecida también ha creado tensiones vecinales.

Una segunda oleada estudentificación está teniendo lugar en los últimos años a la vez que el panorama de la educación universitaria también está cambiando. Con la reciente crisis y política de austeridad, el gobierno liderado por el conservador David Cameron incrementó en el 2011 las tasas universitarias hasta 9.000 libras anuales reemplazando así la subvención estatal a las universidades. A la vez, hemos asistido a un proceso de "yupificación" de la vivienda estudiantil. Este nuevo fenómeno surge en parte en respuesta a las tensiones en los barrios colonizados por estudiantes que se quieren "recuperar" para familias de clase media y en parte por la apertura de un nuevo mercado de vivienda construida especialmente para estudiantes promocionado por inversores privados (Sage et al., 2013). Estos inversores han comprado suelo vacante en las inmediaciones del centro de la ciudad y construido torres de viviendas exclusivas para estudiantes en forma casi de barrios enrejados con sus propias instalaciones deportivas y comerciales ofreciendo una vivencia global estudiantil. Estas nuevas viviendas casi de lujo son mucho más caras y se ofrecen a los estudiantes de clase más alta que cada vez más entienden la educación universitaria como un "paquete comercial" que incluye educación y estilo de vida que les da acceso directo al mercado de trabajo. El efecto inmediato de la construcción de esta especie de hoteles gigantes estudiantiles es el comienzo de un proceso 
de des-estudentificación en los barrios más tradicionales de vivienda estudiantil.

El crecimiento de la población estudiantil en los últimos años también ha tenido un efecto en el paisaje comercial y de consumo del centro de la ciudad. Tal y como argumental Chatterton (2010: 511) "zonas enteras del centro de la ciudad se dedican a servir a los estudiantes, en especial en términos de comercio y ocio. Bares, restaurantes, establecimientos de comida rápida se redirigen para capturar esta población de número considerable, lucrativa y cautiva".

La subida de las tasas universitarias provocó una oleada de protestas y el resurgimiento del movimiento estudiantil en el Reino Unido en el invierno del 2010. En Leeds surgieron también grupos como The Really Open University (Pusey \& Sealey-Huggins, 2013) que pusieron en cuestionamiento no solo la subida de las tasas sino la misma comodificación del conocimiento y la propia institución universitaria. Esta crítica no se extendió, sin embargo, a la comodificación del paisaje urbano estudiantil. Sí que ha existido una oposición más conservadora a la estudentificación en los barrios más tradicionales por la degradación que esta trae a sus barrios

\section{Mixtura social y regeneración urbana}

Antes del estallido de la crisis global financiera y de la llegada del gobierno de corte conservador de David Cameron en mayo de 2010, Leeds estaba atrapada por un cuarto mecanismo de gentrificación, claramente patrocinado por el Estado. Se trata de la regeneración de barrios residenciales con predominio de vivienda social en algunas de las zonas más deprimidas de la ciudad. Aquí nos encontramos de cara con las estrategias bien conocidas de mixtura social (Lees, 2008) en las que cualquier inversión pública del Estado en barrios de vivienda social conlleva casi siempre la rediversificación del régimen de tenencia de la propiedad y la construcción de nueva vivienda privada. Se trata de una estrategia nacional o incluso internacional que tiene también lugar en otras ciudades (Leeds, 2008) y que circula en el "recetario" internacional de políticas urbanas. En el caso del Reino Unido, el Estado central diseña el marco legislativo y financiero de este pro- grama de regeneración urbana e incentiva a los gobiernos locales a competir con otras ciudades por los fondos de regeneración pública o a organizar partenariados públicoprivados y utilizar como financiación la venta de suelo público o recursos económicos de la autoridad local. Generalmente, estos programas están destinados a áreas geográficas específicas que han sido identificadas por el Estado mediante análisis estadístico de acuerdo con determinadas variables como régimen de tenencia, "dependencia" de la población de subsidios del Estado, desempleo, etcétera. El objetivo, a grandes rasgos, es la descomposición de lo que se consideran culturas superhomogéneas de dependencia del Estado concentradas geográficamente en ciertos barrios. Mediante la demolición de vivienda, distribución de la población a otras áreas e introducción de nueva vivienda privada con nuevos residentes se espera mezclar diferentes culturas y estilos de vida que mejoren la calidad de vida de los residentes más pobres y en consecuencia reduzcan su coste económico al Estado. Para las empresas constructoras privadas es un negocio redondo ya que es el Estado quien garantiza los beneficios.

En Leeds se han llevado a cabo dos programas de este tipo de regeneración (Hodkinson, 2011). El primer tipo está dirigido los complejos residenciales de vivienda social (council housing estates) asociados con problemas urbanos en la inner city de Leeds. Según este programa, el ayuntamiento propicia a un consorcio de empresas privadas (bancos, constructoras, inversores) contratos para la regeneración y posterior gestión de las viviendas y sus residentes por un período de 20 o 30 años. Estos programas de remodelación casi siempre incluyen la demolición de parte de la vivienda social y la construcción de nueva vivienda para atraer a nuevos residentes. Algunos de estos barrios como es el caso de Little London en Leeds, están localizados muy cerca del centro de la ciudad y las universidades y el objetivo era el de convertirlos en nuevas zonas de centralidad para residentes profesionales y de clase media. La crisis económica del 2008 ha modificado y retrasado este proyecto nacional gentrificador y parte de los fondos para su financiación han sido cancelados (Hodkinson, 2011). En Leeds, el proyecto de regeneración del barrio de Little London solo se ha llevado a cabo parcialmente. Se han demolido 150 
apartamentos de propiedad pública con la consecuencia del desplazamiento de vecinos dentro y hacia fuera del barrio pero la iniciativa privada no ha construido las 800 nuevas viviendas planificadas por la caída del mercado de la vivienda (Hodkinson, 2011). Nuestra experiencia directa en este barrio confirma que el "fracaso" de este proyecto se debe a las prácticas de resistencia de los vecinos que mediante su escrutinio de los planes, demandas de transparencia y participación y otros tipos de contestación más mundanas consiguieron retrasar el proyecto hasta el momento de la crisis financiera.

El segundo tipo de gentrificación relacionado con la mixtura social se ha implementado en Leeds en un área más amplia que abarcaba la zona este y sur-este de la ciudad, una de las áreas más pobres. Antes de la crisis del 2008 el plan era demoler hasta 3.000 hogares, casi todos de vivienda social, y reemplazarlos con más de 6.000 nuevas viviendas casi todas en régimen de propiedad o alquiler privados. Aquí asistimos a lo que podemos llamar la creación de nuevos mercados de vivienda en zonas que anteriormente estaban bajo el control del Estado. Sin embargo, este proyecto estaba diseñado con una racionalidad precrisis. Se llegaron a demoler unas 300 viviendas pero para cuando se empezaron a construir las nuevas viviendas llegó la crisis del 2008. El ayuntamiento tuvo que rescatar el proyecto comprando algunas de las nuevas viviendas para uso público.

En estos dos programas de mixtura social asistimos a las mismas técnicas de reimaginación de barrios pobres y su mercantilización para la atracción de nuevos residentes de mayor poder adquisitivo. A la vez, se aniquilan prácticas culturales y formas de comunidad de los residentes establecidos. Todo esto se podría interpretar como una forma de "limpieza social" (social cleansing). También se aprecia el interés del Estado por crear brechas en los precios de las rentas urbanas (rent gap) ayudando al capital a explotar zonas urbanas anteriormente no mercantilizadas.

\section{Conclusiones}

En este artículo hemos analizado los procesos de gentrificación en una ciudad del norte de Inglaterra; una ciudad que hemos caracterizado como provincial. Nuestro análisis desvela cuatro mecanismos de gentrificación que atraviesan diferentes temporalidades y escalas geográficas de la ciudad y tienen lugar a través de una multiplicidad de actores, causas y formas. Nuestro argumento es que la oleada actual de gentrificación neoliberal urbana tiene sus bases en las primeras incursiones de constructores privados en los años 80 en las zonas ex industriales cercanas al río y al canal para el desarrollo de vivienda privada destinada a jóvenes profesionales de alto poder adquisitivo; lo que se ha llamado el "city living". Esta transformación urbana tuvo lugar al mismo tiempo que el redescubrimiento y recuperación de las galerías comerciales victorianas en paseos de tiendas de lujo. Poco a poco todo el distrito comercial se ha convertido en una zona de potencial gentrificación. A lo largo de las siguientes dos décadas, esta renovación del centro de Leeds, ha ido atrayendo cada vez a más estudiantes universitarios y contribuyendo a una mercantilización de la vida estudiantil centrada en el consumo de la noche a la vez que a la "colonización" de barrios pobres y residenciales por esta población. Finalmente, hemos sido testigos de un nuevo proceso de gentrificación liderado por el Estado desde el final de los 90 y principios de los 2000 mediante la intervención en barrios residenciales de clase trabajadora a través de demoliciones, renovación, privatización y erección de vivienda nueva bajo la guisa de políticas de mixtura social y regeneración.

En el caso de Leeds una de nuestras principales conclusiones es que los procesos de gentrificación están inacabados, incipientes o incluso fracasados pero a su vez se mantienen como objetivo global de la política urbana local y nacional. La gentrificación es una tendencia que se persigue por la élite política y económica de la ciudad. Como hemos visto, el ayuntamiento, presionado muchas veces por mecanismos de financiación del Estado central, persigue una política de gentrificación del centro de la ciudad y barrios adyacentes. Podemos decir por lo tanto que la gentrificación en Leeds es dirigida por el Estado (state-led). Pero esta política se encuentra con varios límites. Por un lado el propio Estado que no tiene suficiente financiación para llevar a cabo grandes proyectos de rege- 
neración y tiene que recurrir a partenariados con la iniciativa privada. Por otro, esta iniciativa privada solo se implica si ve potencial de obtener plusvalía pero con la llegada de la crisis del 2008 mucha de la iniciativa privada se retiró como hemos visto. Además las grandes empresas nacionales e internacionales solo se interesan por las grandes ciudades y aunque Leeds es una ciudad importante a escala regional no puede competir con Londres u otras ciudades capitales europeas. Otro límite del proyecto gentrificador es también el propio Estado pero desde otro punto de vista. Aunque el estado de bienestar y las políticas sociales están siendo recortadas todavía existen y ponen límites, palían o mitigan en parte los objetivos gentrificadores del gobierno local que actúa a veces de forma contradictoria. Por último está también la contestación. Aunque en Leeds no existen fuertes movimientos urbanos sí que hay una constante contestación de las políticas gentrificadoras del ayuntamiento por parte de asociaciones vecinales y grupos que organizan campañas y eventos que tienen repercusión en los medios locales. Aunque esta contestación no es siempre capaz de parar proyectos por sí misma, al tener lugar a la vez que los otros límites que hemos mencionado, puede tener un efecto de dilación o de disuasión del interés de la iniciativa privada.

En un nivel más teórico, la conclusión a la que hemos llegado al analizar los procesos de gentrificación en Leeds es que hay que contextualizarlos para evitar generalidades y capturar las especificidades de cada ciudad. Así llamamos al análisis de ciertas variables para el estudio de cualquier proceso de gentrificación: escala y posicionamiento, visión relacional de ciudades y políticas urbanas, atención al rol del estado y a la contestación.

\section{Referencias bibliográficas}

BARON, J. Part of historic Leeds city centre building faces demolition. Guardian Leeds Blog. 2010, 20 August. Disponible en internet: http://www.theguardian.com/leeds/2010/ aug/20/first-whit-cloth-hall-demolition-kirkgate

BBC. Architect stands by slums remarks. BBC news channel, 28 January 2009. Dispo- nible en internet: http://news.bbc.co.uk/1/hi/ england/west_yorkshire/7856628.stm

BELL, D. Winter wonderlands: entrepreneurial display, festive socialities and temporary outdoor ice rinks in the UK. Leisure Studies, 2009, No 28, p. 3-18.

BODDY, M. Designer neighbourhoods: new-build residential development in nonmetropolitan UK cities the case of Bristol. Environment and Planning A, 2007, № 39, p. 86-105.

BRENNER, N. \& THEODORE, N. Cities and the geographies of "actually existing neoliberalism". Antipode, 2002, $\mathrm{N}^{\circ} 33$, p. 349-379.

BRENNER, N.; PECK, J. \& THEODORE, $N$. Variegated neoliberalization: geographies, modalities, pathways. Global networks, 2010, № 10, p. 1470-2266.

BRIDGE, G. Time-Space Trajectories in Provincial Gentrification. Urban Studies, 2002, No 40.12 , p. 2545-2556 .

CENTRE FOR CITIES. Cities outlook 2011. London: Centre for Cities, 2011. Disponible en ineternet: http://www.centreforcities.org/ assets/files/Cities\%20Outlook\%202011/ClTIES\%20OUTLOOK_2011.pdf

CHATTERTON, P. The student city: an ongoing story of neoliberalism, gentrification, and commodification. Urban Studies, 2010, № 42, p. 509-14.

CHATTERTON, P. \& HODKINSON, S. Leeds: skyscraper city. The Yorkshire and Humber Regional Review, 2007, Vol. 17, No 1, p. 30-32.

CHATTERTON, P. \& UNSWORTH, R. Making space for culture(s) in Boomtown: some alternative futures for development, ownership and participation in Leeds city centre. Local Economy, 2004, № 19, p. 361-374.

DAVIDSON, M. \& LEES, L. New-build gentrification: its histories, trajectories, and critical geographies. Population, Space, Place, 2010, No 16, p. 395-411. 
DUTTON, P. Leeds calling: the influence of London on the gentrification of regional cities. Urban Studies, 2003, Vol. 12, № 40, p. 2557-2572.

DUTTON, P. The urban renaissance of Leeds: provincial gentrification, a sustainable and equitable urban future? Regeneration and wellbeing: Research into Practice. Bradford: University of Bradford, 2008. Disponible en internet: http://s3.amazonaws.com/zanran_storage/www.bradford.ac.uk/ContentPages/42545630.pdf

EUROSTAT. Regional GDP per capita in the EU in 2010: eight capital regions in the ten first places. Eurostat news release, 2013, $N^{\circ}$ 46. Disponible en internet: http://epp. eurostat.ec.europa.eu/cache/ITY_PUBLIC/1 21032013-AP/EN/1-21032013-AP-EN.PDF

FRIEND OF LEEDS KIRKGATE MARKET. Blog. Disponible en internet: http://kirkgatemarket.wordpress.com/

GONZÁLEZ, S. The north/south divide in Italy and England: discursive construction of regional inequality. European Urban and Regional Studies, 2011, № 18, p. 62-76.

GONZÁLEZ, S. Bilbao and Barcelona 'in motion'. How urban regeneration 'models' travel and mutate in the global flows of policy tourism. Urban Studies, 2012, Vol. 7, No 48 , p. 1397-1418.

GONZÁLEZ, S. \& WALEY, P. Traditional retail markets: the new gentrification frontier? Antipode, 2013, Vol. 4, № 454, p. 965-983.

GREEN, V. Compass. Parallax, 2007, Vol. 1, No 13, p. 9-19.

HARCUP, T. Re-imaging a post-industrial city: the Leeds St Valentine's fair as a civic spectacle. City, 2000, No 4, p. 215-231.

HARRIS, A. From London to Mumbai and back again: gentrification and public policy in comparative perspective. Urban Studies, 2008, Vol. 12 , No 45 , p. 2407-2428.

HAUGHTON, G. Celebrating Leeds as it builds the slums of tomorrow? The Yorkshire and Humber Regional Review, 2009, Vol. 19, $N^{\circ} 2$, p. 28-32.

HAUGHTON, G. \& WILLIAMS, C. (editors) Corporate city? Partnership, participation and partition in urban development in Leeds. Avebury: Ashgate, 1996.

HENDERSON, R. Financial and business services in Leeds: cluster and/or catalyst? The Yorkshire and Humber Regional Review, 2005, Vol. 16, No 4, p. 16-19.

HODKINSON, S. Housing regeneration and the Private Finance Initiative in England: unstitching the neoliberal urban straitjacket. Antipode, 2011, No 43, p. 358-383.

HODKINSON, S. \& CHATTERTON, P. Leeds: an affordable, viable, sustainable, democratic city? The Yorkshire and Humber Regional Review, 2007, Vol. 17, No 2, p. 24-26.

JANOSCHKA, M., SEQUERA, J. \& SALINAS, L. Gentrification in Spain and Latin America - a critical dialogue. International Journal of Urban Regional Research, 2013, Nº 38, p. 1234-1265.

JONES, M. \& WARD, K. Excavating the logic of British urban policy: neoliberalism as the "crisis of crisis-management". Antipode, 2002, Vol. 3, No 34, p. 473-494.

JULIER, G. Design activism meets placebranding: reconfiguring urban representation and everyday practice. In: PIKE, A. Brands and branding geographies. London: Edward Elgar, 2001, p. 213-229.

KOLLEWE, J. Leeds leads the retail way with western Europe's biggest mall project. The Guardian, 2012, 22 of August, s/p. Disponible en Internet: http://www.theguardian. com/business/2012/aug/22/leeds-retail-westen-europe-biggest-mall

LEEDS CITY COUNCIL. 11th city centre audit. Leeds: Leeds City Council, 2013a. Disponible en internet: http://www.leeds.gov.uk/ docs/LEH\%2014\%20City\%20centre.pdf

LEEDS CITY COUNCIL. Leeds monthly economic briefing. Leeds: Leeds City Council, 2013b. Disponible en internet: http://www. 
leeds.gov.uk/docs/LEH\%2001\%20Summary. pdf

LEEDS CITY COUNCIL. Lower Kirkgate planning statement. Draft. Leeds: Leeds City Council, March, 2011b. Disponible en internet: http://www.leeds.gov.uk/Environment_ and_planning/Regeneration/Lower_Kirkgate_Planning_Statement_.aspx

LEEDS CITY COUNCIL. Tall buildings design guide. Supplementary planning document. Leeds: Leeds City Council, 2007. Disponible en internet: http://www.leeds.gov. uk/docs/FPI_TB\%20SPD_012\%20Sustainability\%20Appraisal.pdf

LEEMING, C. Fair exchange? Leeds traders booted out of historic home. Big Issue of the north, 2008, № 703, p. 8-11.

LEES, L. The geography of gentrification. Thinking through comparative urbanism. Progress in Human Geography, 2012, Vol. 2, No 38, p. 155-71.

LEES, L. Gentrification and social mixing: towards an inclusive urban renaissance? Urban Studies, 2008, № 45, p. 2449-2470.

LONDON ECONOMICS. Regional disparities in GDP per capita in the EU after the financial crisis. London: London Economics, 2013. Disponible en internet: http://londoneconomics.co.uk/wp-content/uploads/2013/05/ Regional-disparities-in-GDP-per-capita-inthe-EU-28-05-20131.pdf

LOCATE IN LEEDS. Land Securities' investment is taking Leeds up the league table. Locate in Leeds website, $17^{\text {th }}$ May 2012. Disponible en internet: http://www.locateinleeds. co.uk/2012/05/land-securities-investment-istaking-leeds-up-the-league-table/

MALOUTAS, T. Contextual diversity in gentrification research. Critical Sociology, 2011, № 38, p. 33-48.

MARCUSE, P. Gentrification, abandonment and displacement: connections, causes and policy responses in New York City. Journal of Urban and Contemporary Law, 1985, $\mathrm{N}^{\circ} 28$, p. 195-240.
NATHAN, M \& UNSWORTH, R. Beyond city living: remaking the inner suburbs. Built Environment, 2004, № 32.3, p. 235-249.

O'CONNOR, N.; FLANAGAN, S. \& GILBERT, D. The integration of film-induced tourism and destination branding in Yorkshire, UK. International Journal of Tourism Research, 2008, No 10, p. 423-437.

PECK, J \& THEODORE, N. Mobilizing policy: models, methods, and mutations. Geoforum, 2010, Vol. 2, No 41, p. 169-174.

PUSEY, A. \& SEALEY-HUGGINS, L. Transforming the University: Beyond students and cuts. ACME, Vol. 3, №12, p. 443-458

ROBINSON, J. Urban geography: world cities, or a world of cities. Progress in Human Geography, 2005, Vol. 6, № 29, p. 757-765.

SAGE, J.; SMITH, D. \& HUBBARD, P. New-build studentification: a panacea for balanced communities? Urban Studies, 2013, Vol. 13, NN$^{\circ} 50$, p. 2623-2641.

SHAW, K. Gentrification: what it is, why it is, and what can be done about it. Geography Compass, 2008, № 2, p. 1-32.

SHEPPARD, E. The spaces and times of globalization: place, scale, networks, and positionality. Economic Geography, 2002, Vol. 3, No 78, p. 307-330.

SHEPPARD, E.; LEITNER, E. \& MARINGANTI, A. Provincialising global urbanism: a manifesto. Urban Geography, 2013, Vol. 34, № 7, p. 893-900.

SLATER, T. The eviction of critical perspectives from gentrification research. International Journal of Urban and Regional Research, 2006, Vol. 4, № 30, p. 737-57.

SMITH, D.P. Processes of studentification in Leeds. Report presented to the City and Regional Office. Leeds: University of Leeds, 2002.

SMITH, D. P. 'Studentification': the gentrification factory? In: ATKINSON, R. \& BRIDGE, G. Gentrification in a global context: the 
new urban colonialism. London: Routledge, 2005, p. 72-89.

SMITH, D.P. The buoyancy of 'other' geographies of gentrification: going back-tothe-water and the commodification of marginality. Tijdschrift voor Economische en Sociale Geografie, 2007, № 98, p. 53-67.

SMITH, D.P. The politics of studentification and '(un)balanced' urban populations: lessons for gentrification and sustainable communities? Urban Studies, 2008, Vol. 12, $N^{\circ} 45$, p. 2541-2564.

SMITH, D. P. \& HOLT, L. Studentification and 'apprentice' gentrifiers within Britain's provincial towns and cities: extending the meaning of gentrification. Environment and Planning A, 2007, № 39, p. 142-161.

SMITH, N. New globalism, new urbanism: gentrification as global urban strategy. Antipode, 2002. No 34, p. 427-50.

SPRACKLEN, K.; RICHTER, A., \& SPRACKLEN, B. The eventization of leisure and the strange death of alternative Leeds. City: analysis of urban trends, culture, theory, policy, action, 2013, Vol. 2, No 17, p. 164-178.

STILLWELL, J. \& UNSWORTH, R. Around Leeds: a City Centre Reinvented. Leeds: Leeds University Press, 2008.
TICKELL, A. Taking the initiative: the Leeds financial centre. In: HAUGHTON, G. \& WILLIAMS, C. Corporate City? Partnership, participation and partition in urban development in Leeds. Aldershot: Ashgate, 1993, p. 103-118.

UNWORTH, R. \& SMALES, L. Leeds: shaping change and guiding success. In: PUNTER, J. Urban design and the British urban renaissance. London: Routledge, 2010, p.68-84.

UNSWORTH, R. City living and sustainable development: the experience of a UK regional city. Town Planning Review, 2007, Vol. 6, No 78, p. 725-747.

UNSWORTH, R. City living beyond the boom: Leeds survey 2010. A report by Knight Frank, Morgans City Living, Leeds: Savills, King Sturge and Allsop, 2010. Disponible en internet: http://resources.knightfrank.com/getnewsresource. ashx? $i d=557 a 78 f 1-b c 3 a-4 e 33-$ b3f2-00e83c6270c4\&type =1

WARD, K. The limits to contemporary urban redevelopment: 'doing' entrepreneurial urbanism in Birmingham, Leeds and Manchester. City: analysis of urban trends, culture, theory, policy, action, 2003, Vol. 2, N $N^{\circ} 7$, p. 199-211.

WARD, K. Towards a relational comparative approach to the study of cities. Progress in Human Geography, 2010, Vol. 4, №34, p. 471-487. 
